\title{
The Influence of PEDOT to PSS Ratio on the Optical Properties of PEDOT:PSS Thin Solid Films - Insight from Spectroscopic Ellipsometry
}

\author{
H. Bednarski*, B. Hajduk, J. Jurusik, B. Jarząiek, M. Domański, K. Eaba, A. Wanid
}

AND M. ŁAPKOWSKI

Centre of Polymer and Carbon Materials, Polish Academy of Sciences, M. Curie-Skłodowskiej 34, 41-819 Zabrze, Poland

In this work, we study the influence of the PEDOT to PSS ratio on the optical properties of PEDOT:PSS thin solid films using spectroscopic ellipsometry and UV-vis spectrometry. In the data analysis, we develop a consisted composition dependent optical model of PEDOT:PSS. This enabled us to account for contributions from PSS part within the Tauc-Lorentz optical model and from PEDOT part within the Drude-Lorentz optical model. Moreover, we relate the optical properties of PEDOT:PSS thin solid films to their electrical specific conductivities in the frame of the generalized effective medium theory. Determined in this manner electrical conductivities of five commercially available water dispersions of PEDOT:PSS are compared with their nominal values.

DOI: 10.12693 /APhysPolA.130.1242

PACS/topics: 73.61.Ph, 78.20.Ci, 78.66.Qn

\section{Introduction}

The organic semiconductor poly(3,4-ethylenedioxythiophene) (PEDOT) doped with poly(4-styrenesulfonate) (PSS) has found many different practical applications in organic optoelectronics [1]. It is well known that electrical conductivity of PEDOT:PSS thin solid films depends primarily on the content of conductive PEDOT, whereas PSS acts as doping agent and enables hydration in the form of colloidal dispersion, but simultaneously significantly lowers electrical conductivity of the PEDOT:PSS system [2]. It is thus not astonishing that properties of PEDOT:PSS became an object of intense scientific research, e.g. [1-8] and references therein.

In this work, we report results of our studies on influence of PEDOT to PSS ratio on the optical properties of PEDOT:PSS thin solid films using spectroscopic ellipsometry and UV-vis spectrometry. In optical data analysis, we develop a consisted composition (PEDOT:PSS ratio) dependent optical model of PEDOT:PSS based on the chemical mixture approximation (CMA) [9]. This enabled us to account for contributions from the PSS part within the Tauc-Lorentz optical model and from the PEDOT part within the Drude-Lorentz model. Moreover, we relate the optical conductivity of the PEDOT part to the electrical specific conductivity of PEDOT:PSS in the frame of the generalized effective medium theory (GEMT) [2, 10].

\section{PEDOT:PSS thin films preparation and experimental details}

A series of thin solid films of PEDOT:PSS on glass substrates have been prepared by spin-coating technique.

*corresponding author; e-mail: hbednarski@cmpw-pan.edu.pl
Ground glass (from one side) substrates for ellipsometric characterisation have been used in order to suppress reflections from the back surface. In transmission measurements PEDOT:PSS thin films deposited on quartz substrates have been used. For our study, we have used five $(\mathrm{P} 1 \div \mathrm{P} 5)$ commercially available PEDOT:PSS water dispersions: P1 - PEDOT-PSS high-conductivity grade [Aldrich] 655201, P2 - PEDOT-PSS Clevios HTL Solar [Heraeus], P3 - PEDOT-PSS [Aldrich] 483095, P4 - PEDOT-PSS Al 4083 [Ossila] M121 and P5 PEDOT-PSS low-conductivity grade [Aldrich] 560596. Prior to the thin films deposition the substrates have been cleaned with detergents and then in ultrasonic baths with deionized water, acetone, and isopropanol. After drying, their optical response have been measured using spectroscopic ellipsometer for the purpose of subsequent data analysis. Thin solid films of PEDOT:PSS with different thicknesses have been deposited by varying the spin-coating speed from 5000 to $500 \mathrm{rpm}$. The optical properties of PEDOT:PSS thin solid films were studied within spectroscopic ellipsometer SE Sentech 850 E and their transmission spectra were measured using UV-vis Jasco V-570 spectrometer. For all studied thin films the surface morphology and thickness have been determined using Topometrix Atomic Force Microscope. Additionally, presence of polarons have been confirmed by performing the electron paramagnetic resonance measurement on all used formulations of PEDOT:PSS [8].

\section{Method and approach}

The reflection ellipsometry is based on the fact that linearly polarized light reflected from surface changes polarization to the elliptical. In the case of materials either optically isotropic or with uniaxial anisotropy no mixing of $p$ and $s$ polarized components of light waves upon reflection occurs. Therefore, the standard reflection ellipsometry is sufficient to study optical response of the 
spin-coated thin solid films of PEDOT:PSS, which reveal uniaxial optical anisotropy [7]. Ellipsometry measures the complex reflectance ratio $\rho=\mathrm{e}^{\mathrm{i} \Delta} \tan \Psi$, where $\Psi$ and $\Delta$ are the ellipsometric angles. On the other hand, $\rho$ depends on the complex dielectric functions of a particular optical system and must be determined theoretically.

In our studies, the thin solid film of PEDOT:PSS is deposited on a glass substrate, thus corresponding optical system consist of the ambient air, the thin solid film and the glass substrate. Now, we are building an optical model for the composite material, like PEDOT:PSS, which combines properties of its two constituents and is composition dependent. For this purpose, we use CMA. In this approach, the complex dielectric function of the composite material $\varepsilon$ is expressed by a sum of weighted contributions, as follows [9]:

$$
\varepsilon(f)=f \varepsilon_{I}(f)+(1-f) \varepsilon_{H}(f),
$$

where $\varepsilon_{H}$ is the dielectric function of a majority material, $\varepsilon_{I}$ is the dielectric function of a minority material (inclusion) and $f$ is the volume fraction of the inclusion phase. In the case of PEDOT:PSS water dispersions, the hygroscopic PSS is always the majority material to ensure their temporal stability. Moreover, PSS acts as a doping agent, and both the PEDOT oligomers and the PSS long chains interact strongly electrostatically forming the PEDOT:PSS complex. This fact supports application of the CMA to the PEDOT:PSS system. Practically, we have assumed $\varepsilon_{H}(f)=\varepsilon_{\mathrm{PSS}}$, i.e. that $\varepsilon_{\mathrm{PSS}}$ is independent of $f$, and that $\varepsilon_{\text {PEDOT }}(f)$ can be described by the DrudeLorentz optical model. In analysis of the optical data we have used the computer software SpectractraRay $/ 3$ from SENTECH Instruments [9].

Now, we are relating the optical properties of PEDOT:PSS thin solid films to their specific electrical conductivity. The corresponding, static, specific conductivity $\sigma$ can be determined from the Drude model parameters as follows:

$$
\sigma=\frac{\omega_{p}^{2}}{\omega_{\tau}}
$$

where, $\omega_{p}$ and $\omega_{\tau}$ are the Drude-oscillator strength and its damping parameter, respectively. Obtained in this manner optical conductivity, with parameters $\omega_{p}$ and $\omega_{\tau}$ determined within our optical model of PEDOT:PSS, corresponds to that of PEDOT. Therefore, in order to calculate the corresponding electrical conductivity of PEDOT:PSS we apply GEMT. In Ref. [6], GEMT has been successfully applied to description of the composition dependent specific electrical conductivity of PEDOT:PSS. The GEMT relates specific electrical conductivities of a conductive inclusion material $\left(\sigma_{c}\right)$ and more resistive matrix material $\left(\sigma_{r}\right)$ with the conductivity of a composite media $\left(\sigma_{m}\right)$ by the following equation $[2,10]$ :

$$
\frac{(1-f)\left(\sigma_{r}^{1 / w}-\sigma_{m}^{1 / w}\right)}{\sigma_{m}^{1 / w}+\frac{1-f c}{f c} \sigma_{m}^{1 / w}}+\frac{f\left(\sigma_{c}^{1 / w}-\sigma_{m}^{1 / w}\right)}{\sigma_{c}^{1 / w}+\frac{1-f c}{f c} \sigma_{m}^{1 / w}}=0,
$$

where $f$ is the volume fraction of the conductive phase, $f_{c}$ is the (critical) percolation volume fraction of the inclusion material and the model parameter $w$ denotes the critical exponent. Usually, $w$ is adjusted to the experimental data $[2,10]$.

While describing our approach within Eqs. (1)-(3), we do not take under consideration materials anisotropy. Spin-coated PEDOT:PSS thin solid films reveal uniaxial anisotropy with the optical axis perpendicular to the surface, so such an extension should be shortly outlined. Generally, the dielectric function is a 3D tensor quantity. In the case of the PEDOT:PSS films, this tensor is diagonal and may be described by its two components. Namely, the ordinary $\varepsilon_{\|} \equiv \varepsilon_{x} \equiv \varepsilon_{y}$ component and extraordinary components $\varepsilon_{\perp} \equiv \varepsilon_{z}$. Accordingly, Eqs. (1)(3) should be additionally labelled for each component \| and $\perp$ without any changes in their functional form.

\section{Results and discussion}

According to Eq. (1), composition dependent dielectric function $\varepsilon(f)$ of PEDOT:PSS consists of the weighted sum of contributions from PEDOT and PSS. As we found, it is well represented by the three Tauc-Lorentz oscillators, which describes three absorption bands of PSS in the high phonon-energy region. Both complex components of $\varepsilon_{\mathrm{PEDOT}}(f)$, the ordinary and the extraordinary one, are described by the Drude-Lorentz optical model with the three Lorentz oscillators. Determined in such a manner $\varepsilon(f)$ constitutes our optical model of PEDOT:PSS thin solid films and has been fitted to our ellipsometric data.

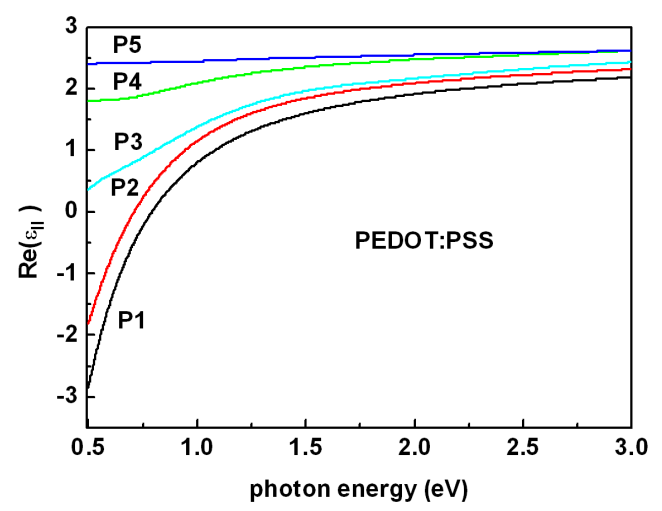

Fig. 1. Comparison of the real components of ordinary dielectric functions, for five studied formulations of PEDOT:PSS (P1-P5), as obtained from ellipsometric data analysis within our composition dependent optical model of PEDOT:PSS.

In Fig. 1 we compare real components of PEDOT:PSS $\varepsilon_{\|}$at phonon energy in the range of $0.5-3 \mathrm{eV}$ and for different PEDOT contents. One can observe from Fig. 1 that the $\operatorname{Re}\left(\varepsilon_{\|}(f)\right)$ increases systematically for decreasing PEDOT content $f$, i.e. from $\mathrm{P} 1$ to $\mathrm{P} 5$. Thus, as can be expected, Fig. 1 reveals improvement of the chargecarriers conduction in PEDOT:PSS thin solid films with increase of $f$. To illuminate more this aspect, we present in Table I calculated values of the compositions $f$ and optical specific conductivities, for five studied water 
TABLE I

Comparison of calculated compositions $(f)$ and optical conductivities (in plane $\sigma_{\|}$and out of plane $\sigma_{\perp}$ ) for PEDOT:PSS thin solid films with corresponding nominal values $\sigma_{N}$ of five studied PEDOT:PSS water dispersions $(\mathrm{P} 1-\mathrm{P} 5)$

\begin{tabular}{c|c|c|c|c|c}
\hline \hline $\begin{array}{c}\text { PEDOT: } \\
\text { PSS }\end{array}$ & $\begin{array}{c}\text { PEDOT } \\
\text { to PSS }\end{array}$ & $\begin{array}{c}\sigma_{N} \\
{\left[\mathrm{~S} \mathrm{~cm}^{-1}\right]}\end{array}$ & $f$ & $\begin{array}{c}\sigma_{\|} \\
{\left[\mathrm{S} \mathrm{cm}^{-1}\right]}\end{array}$ & $\begin{array}{c}\sigma_{\perp} \\
{\left[\mathrm{S} \mathrm{cm}^{-1}\right]}\end{array}$ \\
\hline P1 & $1: 2.5$ & 150 & 0.40 & 2453 & 76.8 \\
P2 & $0.5 \%: 0.8 \%$ & 1.0 & 0.29 & 2335 & 102.9 \\
P3 & - & $0.1 \div 1.0$ & 0.30 & 3808 & 56.8 \\
P4 & $1: 6$ & $2 \times 10^{-3} \div 10^{-4}$ & 0.18 & 252.7 & 0.28 \\
P5 & $0.14 \%: 2.6 \%$ & $10^{-5}$ & 0.08 & 65.2 & $7 \times 10^{-4}$
\end{tabular}

dispersions of PEDOT:PSS. Additionally, for the sake of comparison, the corresponding nominal values of the composition and specific conductivity were also provided in Table I. Comparison of the optical conductivity values reveals their strong dependence on PEDOT content and this is in line with observed tendency in Fig. 1. However, comparison of the nominal electrical conductivities with determined using the Drude oscillator parameters shows very large differences. Reasons may be numerous. Firstly, this difference may result from complex hierarchical microscopic structure of PEDOT:PSS. Namely, the hydrophilic nature of PSS chains and the hydrophobic nature of PEDOT oligomers result in formation of micelles in the water solution. Numerous studies lead to rather common picture that the PEDOT:PSS micelles have a core-shell structure in which the core is rich in PEDOT oligomers and is surrounded by PSS rich shell. Therefore, optical techniques probe rather local conductivity in PEDOT rich regions, whereas electrical measurements give global conductivity to which contribute much more resistive PSS. Secondly, within our approach we have determined optical conductivity of the PEDOT part which is certainly larger than that of PEDOT:PSS. For these reasons, we have applied GEMT, i.e. Eq. (3), to estimate the electrical conductivity of studied here thin solid films of the different PEDOT:PSS dispersions.

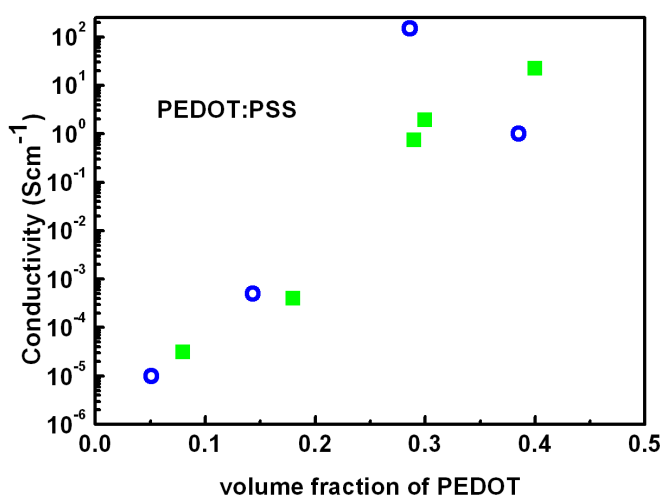

Fig. 2. Comparison of the composition $f$ dependent electrical in plane conductivity $\sigma_{m}$, calculated using Eq. (3) and $\sigma_{c}$ determined within our optical model of PEDOT:PSS (filled symbols), with the nominal conductivity $\sigma_{N}$ of corresponding water dispersions of PEDOT:PPS at their nominal compositions (empty symbols) estimated from the PEDOT to PSS ratio.
Results of these calculations for in plane conductivity are presented in Fig. 2. For the purpose of comparison, in Fig. 2 we have drawn also the corresponding nominal values of the electrical conductivity, open symbols. Now, agreement is significantly better. The GEMT calculations have been performed using following set of parameter values: $f_{c}=0.245, \sigma_{r}=0.00001 \mathrm{~S} \mathrm{~cm}^{-1}$ and $w=3$. It should be noted that as indicated by Meredith et al. [11] for oblate spheroids following condition is fulfilled $0.1077>f_{c} / w>0$ and that corresponding value determined by us is 0.0817 .

\section{Conclusions}

We have studied the influence of the PEDOT to PSS ratio on the optical properties of PEDOT:PSS thin solid films using spectroscopic ellipsometry and UV-vis spectrometry. We have developed a consisted composition dependent optical model of PEDOT:PSS based on the chemical mixture approximation. This enabled us to account for contributions from PSS part within the TaucLorentz optical model and from PEDOT part within the Drude-Lorentz model. Moreover, we have related the optical conductivity of the PEDOT part to the electrical specific conductivity of PEDOT:PSS in the frame of the generalized effective medium theory.

\section{Acknowledgments}

This work was supported by the NCN grant (Poland) No. DEC-2013/09/B/ST8/01629

\section{References}

[1] W. Lövenich, Polym. Sci. Ser. C 56, 135 (2014).

[2] T. Stöcker, A. Köhler, R. Moos, J. Polym. Sci. Part B Polym. Phys. 50, 976 (2012).

[3] S.-I. Na, G. Wang, S.-S. Kim, T.-W. Kim, S.-H. Oh, B.-K. Yu, T. Lee, D.-Y. Kim, J. Mater. Chem. 19 9045 (2009).

[4] F. Herrmann, S. Engmann, M. Presselt, H. Hoppe, S. Shokhovets, G. Gobsch, Appl. Phys. Lett. 100, 153301 (2012).

[5] A.M. Nardes, M. Kemerink, R.A.J. Janssen, J.A.M. Bastiaansen, N.M. Kiggen, B.M.W. Langeveld, J.J.M. v. Breemen, M.M. de Kok, Adv. Mater. 19, 1196 (2007).

[6] U. Lang, E. Müller, N. Naujoks, J. Dual, Adv. Funct. Mater. 19, 1215 (2009).

[7] L.A.A. Pettersson, S. Ghosh, O. Inganäs, Org. Electron. 3, 143 (2002).

[8] P. Zassowski, W. Domagala, M. Lapkowski, P.J. Skabara, Key Eng. Mater. 559, 121 (2013).

[9] U. Richter, SpectraRay/3 Software Manual, Sentech Instruments GmbH, 2011.

[10] R.H. Cruz-Estrada, M.J. Folkes, J. Mater. Sci. Lett. 21, 1431 (2002).

[11] R.E. Meredith, C.W. Tobias, in: Advances in Electrochemistry and Electrochemical Engineering, Vol. 2, Ed. C.W. Tobias, Interscience, New York 1962, p. 15. 